\title{
Erratum to: Comparative quantitative study of 'signature' pathological lesions in the hippocampus and adjacent gyri of 12 neurodegenerative disorders
}

\author{
Richard A. Armstrong ${ }^{1}$ Nigel J. Cairns ${ }^{2,3}$
}

Published online: 11 June 2015

(C) Springer-Verlag Wien 2015

\section{Erratum to: J Neural Transm}

DOI 10.1007/s00702-015-1402-8

Unfortunately, the online published article has errors in Table 1. The correct table is given in the following page.

The online version of the original article can be found under doi:10.1007/s00702-015-1402-8.

Richard A. Armstrong

R.A.Armstrong@aston.ac.uk

1 Vision Sciences, Aston University, Birmingham B4 7ET, UK

2 Department of Neurology, Washington University School of Medicine, Saint Louis, MO 63110, USA

3 Department Pathology and Immunology, Washington University School of Medicine, Saint Louis, MO 63110, USA 
Table 1 Summary of demographic details, signature pathology, associated pathology, and diagnostic criteria in the disorders studied

\begin{tabular}{lllllll}
\hline Disorder & $N$ & Mean age (SD) & M:F & Signature lesion & Additional pathology & Diagnostic criteria \\
\hline AD & 20 & $76(13.0)$ & $5: 15$ & $\mathrm{~A} \beta$ deposits NFT & EN, GVC & NINCDS/ADRDA/CERAD \\
sCJD & 11 & $67(8.5)$ & $6: 6$ & PrP $^{\text {sc }}$ deposits & Vacuolation & Budka et al. (1995) \\
vCJD & 11 & $29(9.1)$ & $5: 6$ & PrP $^{\text {sc }}$ deposits & Vacuolation & Ironside et al. (2000) \\
DS & 12 & $51(9.7)$ & $6: 6$ & $\mathrm{~A} \beta$ deposits & NFT & By karyotype \\
PD-Dem & 15 & $75(5.0)$ & $12: 3$ & LB & LN, LG & UKPDSBB \\
PiD & 10 & $65.3(11.3)$ & $7: 3$ & PB & NFT, PC & Cairns et al. (2007) \\
CBD & 12 & $64.7(9.07)$ & $8: 4$ & NCI & AP, GI, EN & NIH-ORD \\
PSP & 8 & $73.4(7.4)$ & $4: 4$ & NFT & TA, GI, NP & NINDS-SPSP \\
DLB & 12 & $73.8(7.2)$ & $12: 0$ & LB & DN & CDLB \\
MSA & 10 & $66.5(8.51)$ & $7: 3$ & GCI & NCI & GI, DN \\
NIFID & 10 & $45.3(12.1)$ & $7: 3$ & NCI & NII, DN, GI & Cairns et al. (2007) \\
FTLD-TDP & 15 & $69.6(8.50)$ & $7: 2$ & NCI & Cairns et al. (2007) \\
\hline
\end{tabular}

Diagnostic criteria: 'National Institute of Neurological and Communicative Disorders and Stroke and the Alzheimer Disease and Related Disorders Association' (NINCDS/ADRDA) (Tierney et al. 1988), 'Consortium to Establish a Registry of Alzheimer Disease' (CERAD) criteria (Mirra et al. 1991); United Kingdom Parkinson Disease Society Brain Bank clinical diagnostic criteria (UKPDSBB); National Institute of Health-Office of rare disorders (NIH-ORD); National Institute of Neurological Disorders and Stroke (NINDS) and the Society of PSP (SPSP) (NINDS-SPSP) (Hauw et al. 1994; Litvan et al. 1996a, b); 'Consortium on Dementia with Lewy bodies' (CDLB)' (McKeith et al. 1996); 'Minneapolis Consensus Criteria (MCC) (Gilman et al. 1998); diagnostic criteria for PiD, NIFID (FTLD-FUS) and FTLD-TDP according to Cairns et al. (2007)

Disorders: $A D$ Alzheimer's disease, $D S$ Down's syndrome, $P i D$ Pick's disease, $C B D$ corticobasal degeneration, $P S P$ progressive supranuclear palsy, $D L B$ dementia with Lewy bodies, MSA multiple system atrophy, NIFID neuronal intermediate filament inclusion disease, FTLD-TDP frontotemporal lobar degeneration with TDP-43 proteinopathy, PD-Dem Parkinson's disease dementia, $s C J D$ sporadic Creutzfeldt-Jakob disease, $v C J D$ variant Creutzfeldt-Jakob disease

Neuropathology: $A P$ astrocytic plaques, $D N$ dystrophic neurites, $E N$ enlarged neurons, $G I$ glial inclusions, $G V C$ granulovacuolar change, $L G$ Lewy grains, $L N$ Lewy neurites, NCI neuronal cytoplasmic inclusions, NFT neurofibrillary tangles, NII neuronal internuclear inclusion, $P C$ Pick cells

$N$ number of cases studied, $M$ male, $F$ female, $S D$ standard deviation 